\title{
Kräuterbücher (4)
}

Tulpen in den Prachtgärten der Kaufleute und Fürsten des 16. bis 18. Jahrhunderts: Erlesene Kostbarkeiten zu ruinösen Preisen

Barbara Brauckmann

Nachdem jahrhundertelang in Klostergärten medizinisch verwendbare Heilkräuter den Vorrang hatten und in mitteleuropäischen Grünanlagen einheimisches Saatgut dominierte, begannen im 16. Jahrhundert Herrscher und wohlhabende Patrizier aus Handelsverbindungen mit Südosteuropa oder Kleinasien bezogene exotische Gewächse zu kultivieren.

$\mathrm{Zu}$ den beliebtesten damaligen Raritäten gehörte die Tulpe, die bereits Conrad Gessner (1516-1565) (siehe auch Beitrag in Heft 3/14) im April 1559 bei einem Besuch im botanischen Privatgarten des reich gewordenen Augsburger Kaufmanns und Ratsherrn Heinrich Herwarts (15201583) abzeichnete und als rote Lilie (Tulipa turcarum) beschrieb [1].

Die ursprünglich aus den Steppen Zentralasiens stammende, Lâle genannte rote Blume gelangte über die Gärten am persischen Hof in Isfahan nach Istanbul. Zur Verbreitung der Tulpe nach Europa trug der flandrische Diplomat Ogier Ghislain de Busbecq (1520-1592) massgeblich bei, der als Gesandter des österreichischen Kaisers Ferdinand I. im Herbst 1554 zu Süleyman I. nach Konstantinopel reiste, um einen Waffenstillstand mit dem Osmanischen Reich auszuhandeln [2]. Dort erhielt er vom Sultan zunächst keine Audienz, aber einige Fliederpflanzen sowie Hyazinthen- und Tulpenzwiebeln. Letztere nannte er 1554 in einem Brief Tulipam und schickte Exemplare an seinen Freund Carolus Clusius (Charles de l'Écluse; ${ }^{\star} 1526$ in Arras, $\dagger 1609$ in Leiden) in Wien, einem bedeutenden Botaniker und Gärtner mit einer Vorliebe für orientalische Zwiebelpflanzen [3].

Jener Clusius hatte Jura in Marburg, im Jahr 1549 Philosophie bei Philipp Melanchthon in Wittenberg und wenige Jahre später Medizin und dann Botanik in Montpellier studiert. 1554 zog er über die Schweiz nach Antwerpen, wohin sein Vater als Calvinist flüchten musste. Von hier aus unternahm er weite Reisen nach West- und Südeuropa, unter anderem 1564 mit Jakob III. Fugger (1542-
1598) nach Spanien und Portugal. Sein Werk «Rariorum aliquot stirpium per Hispanias observatorum Historia» von 1576 enthielt einen Anhang, in dem er verschiedene Zwiebelblumen aus der Türkei darstellte, insbesondere Tulpen.

Im Jahr 1573 erreichte ihn durch Vermittlung unter anderem von Busbecq die Einladung des Kaisers Maximilian II. (1527-1576), in Wien einen botanischen Garten anzulegen und zu betreuen. Dort wohnte Clusius bei dem vermögenden Dr. Johann Aichholtz (1520-1588), einem ebenfalls an Reisen und Pflanzen interessierten Hofmedicus und Anatomieprofessor. Doch schon 2 Jahre nach seiner Ankunft starb der Kaiser, und sein 24 Jahre alter, der Botanik gleichgültig gegenüberstehender Sohn $\mathrm{Ru}-$ dolf II. bestieg den Thron. Der katholisch erzogene Herrscher zahlte dem Calvinisten Clusius monatelang kein Gehalt mehr und entliess ihn 1577 insbesondere wegen seines Glaubens. Dennoch blieb der Gelehrte noch viele Jahre in Wien.

Zur Stimmungsaufhellung in dieser Zeit trugen die Einladungen des Freiherrn und Generals Balthasar III. von Batthyány (1543-1590) nach Güssing bei, wo Clusius dessen von türkischen Gefangenen erworbene Samen, Wurzeln und Zwiebeln aus Konstantinopel studieren durfte [3].

Immer wieder musste der Botaniker Rückschläge erdulden. Einerseits, wenn ihm Strassendiebe oder Bedienstete wertvolles Saatgut stahlen, andererseits, wenn seine Kulturen durch Kälte oder Nässe verdarben. Im Dezember 1586 erlitt er zudem einen Schenkelbruch, der ihn etliche Zeit am Gehen hinderte.

Schliesslich übersiedelte er 1588 nach Frankfurt am Main, wo ihm Landgraf Wilhelm IV. von Hessen (Amtszeit 1567-1592) ein Jahresgehalt bezahlte, sodass er 1589 im eigenen Garten neben Rosskastanie und orientalischen Zwiebelpflanzen auch die ersten Kartoffeln anpflanzen konnte, die damals als Heil- und Zierpflanzen

\section{KARGER \\ Fax +497614520714 Information@Karger.com} www.karger.com
Dr. Barbara Brauckmann

Öffentlichkeitsarbeit, Departement Chemie und Angewandte Biowissenschaften ETH Zürich

HCI J 57.2, Vladimir-Prelog-Weg 10, 8093 Zürich, Schweiz

brauckmann@chem.ethz.ch 
Abb. 1. Titelblatt von «Caroli Clusi Atrebatis Rariorum Plantarum Historia quae accesserint, proxima pagina docebit», Ex officina Plantiniana, Antverpia 1601 (Foto: Departement Chemie und Angewandte Biowissenschaften der ETH Zürich).

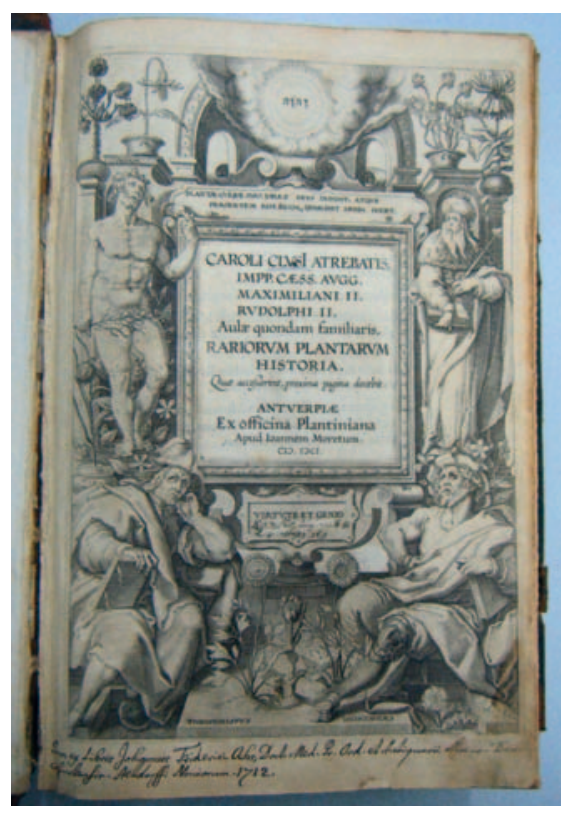

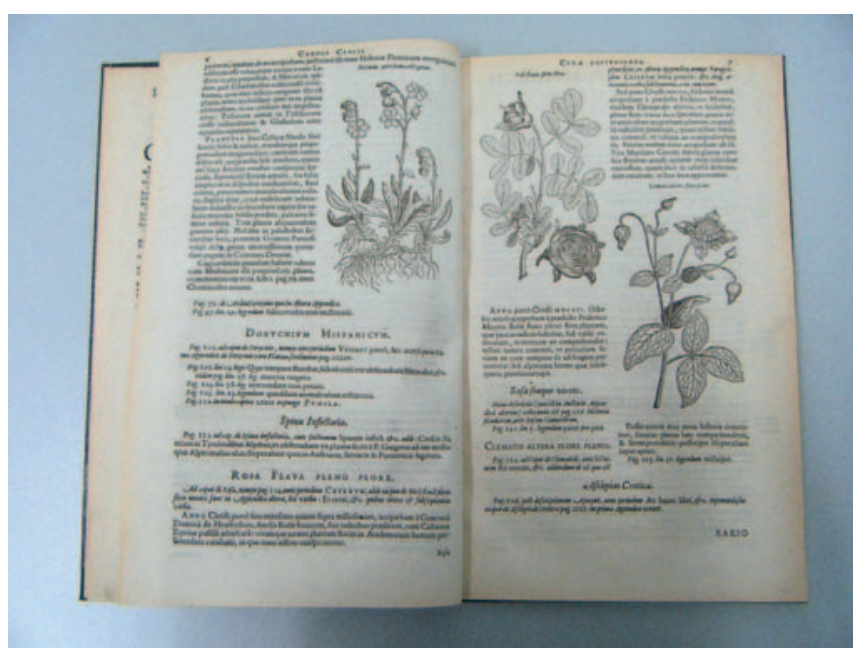

Abb. 2. "Caroli Clusi Atrebatis Rariorum Plantarum Historia quae accesserint, proxima pagina docebit»: Seiten mit verschiedenen Pflanzen wie Rose und Clematis (Foto: Departement Chemie und Angewandte Biowissenschaften der ETH Zürich). galten. Philippe de Sivry, der Gouverneur von Mons (Belgien), hatte ihm zwei Taratoufflo genannte Kartoffelknollen als Geschenk zukommen lassen.

Durch einen Treppensturz verrenkte sich Clusius bald noch die Hüfte und war von nun an auf Krücken angewiesen. Das Angebot auf eine Botanikprofessur an der Universität bewog ihn, im Oktober 1593 nach Leiden zu übersiedeln. Mit etlichen Tulpen im Gepäck übernahm er die Errichtung und Leitung des Hortus Academicus, ohne Verpflichtung, Botanikunterricht zu erteilen. Ihm gelangen aufwendige Kreuzungen und Aussaatversuche mit verschiedenen Pflanzenarten, die er gemäss ihrer genauen Form charakterisierte. Zudem versuchte er sie mit zwei kurzen Wörtern zu benennen, ähnlich der später von Carl von Linné entwickelten binären Nomenklatur.

Als ihm 1596 wieder einmal über Hundert seiner kostbaren Saatzwiebeln aus dem Garten geraubt wurden, löste er ihn frustriert auf. Tulpenzwiebeln genoss er zwar weiterhin gern in Zucker eingelegt, widmete sich von nun aber wieder vermehrt dem Briefwechsel und seinen Publikationen: 1601 erschien das Werk «Rariorum plantarum historia» (Abb. 1, 2) und 1605 das «Compendium Exoticorum libri decem».

\section{Zunächst Tauschgeschäfte, dann Auktionen und «Windhandel»}

Einige Jahre später begannen die niederländischen Kaufleute mit Tulpenzwiebeln zu handeln, wenn auch zunächst nur während der Pflanzzeit. Als allmählich die Nachfrage stieg, das Angebot aber konstant blieb, begann das Risikogeschäft [4]. An die Interessenten wurden auch
Zwiebeln verkauft, die sich noch in der Erde befanden und erst nach der Blüte ausgegraben werden konnten. Reichte das Geld für eine ganze Zwiebel nicht, erwarb der einfachere Bauer vielleicht nur einen Gewichtsanteil davon. Da die mehrfarbigen Blumen höhere Gewinne ermöglichten, begannen Betrüger, einfache Tulpen als kostbare Exemplare mit gemusterten Blüten auszugeben. Eine Zwiebel der seltenen «Semper Augustus» mit blauem Blütenboden und roten «Flammen» auf weissen Blättern erreichte 1633 umgerechnet einen Rekordpreis von 10000 Gulden, wofür es auch ein edles Amsterdamer Stadthaus in bester Grachtenlage gegeben hätte. Die fast genauso schöne Sorte «Viceroy» wurde ebenfalls gegen Schiffe, Erntegüter oder Haushaltsinventar getauscht. Die begehrten geflammten Farbverläufe, Streifen oder Flecken liessen sich nämlich weder züchten noch künstlich mit Mörtelstaub, Taubenkot oder sonstigen Zutaten erzielen. Damals konnte niemand wissen, dass für die ersehnte Musterung das Tulpenmosaik-Virus in den Gärten verantwortlich war, das die gleichmässige Verteilung des Pflanzenfarbstoffs Anthozyan im Blütenblatt auf kleinere Bereiche begrenzt. Nachdem etliche Mühlen, Brauereien und andere Lebensgrundlagen verspekuliert worden waren und selbst Rembrandt van Rijn (1606-1669) sein Vermögen verkalkuliert hatte, platzte im Februar 1637 schliesslich in den Niederlanden die «Tulpenblase» [5].

\section{Farbenpracht in Garten und Buchwerk: Der Hortus Eystettensis}

Clusius gab zwar immer noch Geld zur Beschaffung von Pflanzen aus, tauschte sie ansonsten jedoch eher ein 


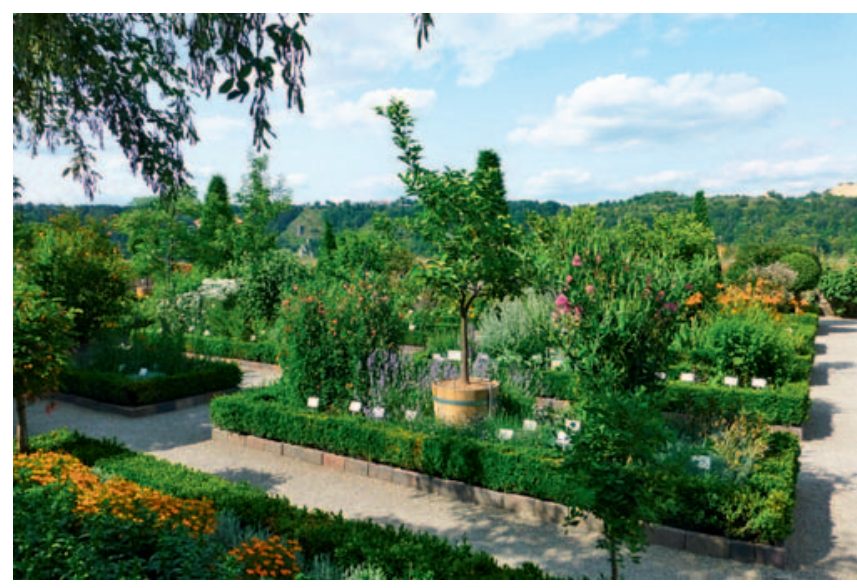

Abb. 3. Der Bastionsgarten in Eichstätt mit der Pflanzenwelt des Kupferstichwerkes «Hortus Eystettensis» im Juli 2013 (Foto: Barbara Brauckmann).

oder verschenkte sie an Bekannte und Freunde. $\mathrm{Zu}$ diesen gehörte auch Joachim Camerarius II. (1534-1598 in Nürnberg [3]; der Sohn des Philologen und Historikers Joachim I.), der nach einem Medizinstudium in Padua (1559-1562) und Bologna (1564) die Stelle eines Stadtarztes in Nürnberg angetreten hatte. Dieser hatte dank seines Vermögens den Nachlass von Conrad Gessner kaufen können und 1586 das «Kreuterbuch» von Pietro Andrea Matthioli (1500-1577) übersetzt.

Mit Clusius verband ihn ein über 25 Jahre währender Briefwechsel. Beide stellten eine grosse Menge an Pflanzenmaterial für den im Umbau befindlichen botanischen Garten des Tulpensammlers Johann Conrad von Gemmingen (1561-1612) [6] zur Verfügung.

Der Fürstbischof von Eichstätt ermächtigte nämlich Camerarius, auf dem Areal seiner Residenz Willibaldsburg in Eichstätt einen Renaissance-Lustgarten zu errichten, weil dieser bereits den Landgrafen Wilhelm IV. in Kassel beim Anlegen eines solchen Repräsentationsobjektes beraten und ausserdem in Nürnberg den ersten wissenschaftlich geordneten botanischen Garten angelegt hatte.

Nach dem unerwarteten Tod von Camerarius im Jahr 1598 ging der Auftrag an den Nürnberger Apotheker Basil Besler (1561-1629) [7] über, der den Hortus Eystettensis [8] mit acht einzelnen Gartenbereichen und einem Springbrunnen vollendete. (Ein solcher Garten (Abb. 3) wurde 1998 auf der Schmiedebastion der Willibaldsburg mit den gleichen Pflanzen nachgebaut.)

Besler überzeugte den Fürstbischof bald von der Idee einer Buchpublikation zu den Gartenpflanzen. Da von Gemmingen für den kunstvoll gestalteten Pflanzenatlas vom neuen Druckverfahren des Kupferstichs profitieren wollte, organisierte Besler hierfür Zeichner, Drucker und Kupferstecher und überwachte die Arbeiten. Von Anfang an waren zwei unterschiedliche Ausgaben vorgesehen:
Die kostbare, einseitig bedruckte, meist kolorierte und 14 kg schwere Ausgabe $(56 \times 47 \times 13 \mathrm{~cm})$ in kleiner Stückzahl sollte als fürstliches Geschenk dienen und kostete rund 500 Gulden. Die billigere «Buchhandelsausgabe» mit einer Auflage von etwa 300 Exemplaren für 35-48 Gulden war für das Fachpublikum vorgesehen (Abb. 4). Bis zu seinem Tod 1612 hatte der Fürstbischof 7500 Gulden für die Herstellung aufgewandt; die Gesamtkosten für die fürstbischöfliche Kasse beliefen sich später auf 18000 Gulden [8]. Und Besler konnte sich 1616 für 2500 Gulden ein stattliches Haus in Nürnberg leisten.

Von Gemmingen ging es bei Garten und Buch um fürstliche Repräsentation und nicht um botanische Wissenschaft. Zu jeder Buchtafel gab es daher lediglich Hinweise auf Fundstellen in der botanischen Fachliteratur von unter anderem Camerarius, Clusius, Bauhin, Fuchs, Tabernaemontanus und Lobelius sowie die Angabe der gebräuchlichen deutschen Namensform. Das Werk ist in vier Jahreszeitengruppen gegliedert, mit je 7-18 Tafeln, auf denen sich jeweils bis zu 6 Einzelpflanzen finden. Diese Idee stammte vermutlich noch von Camerarius. Dessen Neffe, der Altdorfer Botanikprofessor Ludwig Jungermann, lieferte die kurzen lateinischen Texte [9].

Das später mehrfach wieder aufgelegte und nicht allzu erfolgreiche Werk zeigt 349 deutsche, 209 süd- und südosteuropäische, 63 asiatische, 9 afrikanische und 23 amerikanische Arten, zumeist in natürlicher Grösse. Es finden sich Farbillustrationen von Kaiserkrone, Artischocke, Kürbis, Paprika, Sonnenblumen und Tomaten. Besonders an der grossblütigen, von Clusius auch in «Rariorum plantarum historia» beschriebenen Kartoffel («Papas Peruanorum») lässt sich ermessen, dass es sich hier zunächst um eine Zierpflanze handelte. Von den rund 1000 Einzelabbildungen der Arznei-, Nutz- und Zierpflanzen des Gartens gelten nur 653 als wirkliche Spezies. Viele Seiten zeigen Bilder mit prächtig gefärbten Tulpen (Abb. 5) [10].

\section{Tulpenbücher gegen das Verwelken und Vergessen}

Unabhängig vom «Börsencrash», dessen Thematik noch Jahrhunderte später Schriftsteller zu Romanen inspirierte (z.B. «Adrian der Tulpendieb» von Otto Rombach oder «La Tulipe noire» von Alexandre Dumas (18021870)), blieben Tulpen weiterhin beliebte und kostbare Frühlingsblüher in den Gärten der Wohlhabenden. Ein bekannter fürstlicher Tulpenliebhaber war Karl III. Wilhelm von Baden-Durlach ( ${ }^{*} 1679$ in Durlach; $† 1738$ in Karlsruhe - inmitten seiner Blumenbeete) [11], der 1715 die Stadt Karlsruhe gründete und die neue Residenz dorthin verlegte. Die Blumen für seine Gärten bezog er hauptsächlich aus Holland, wo er beispielsweise im Sommer 1723 mit grossem Gefolge zur «Gemüthsergötzung» 4 


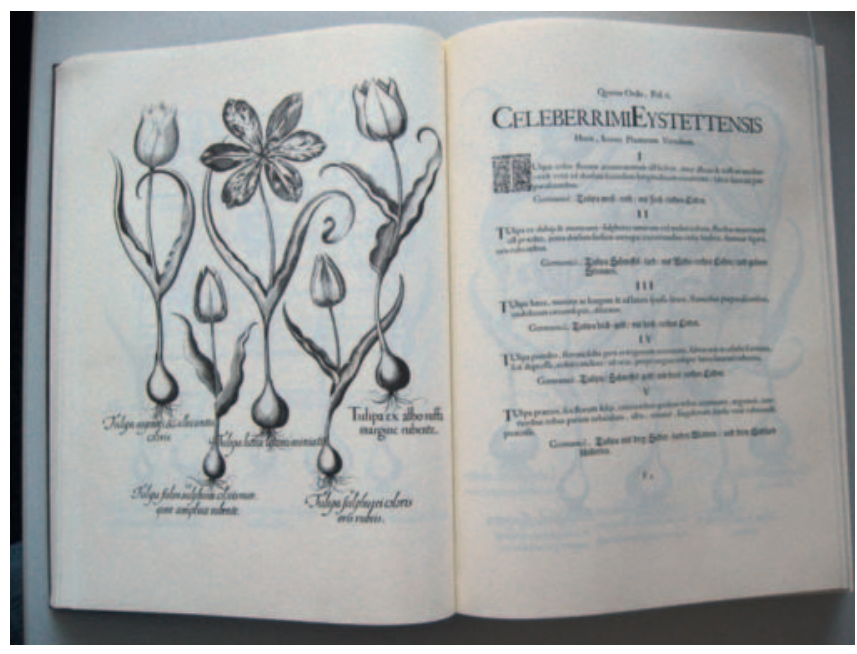

Abb. 4. Basilius Besler: «Hortus Eystettensis sive diligens et accurata omnium plantarum...». Reprint der Ausgabe von 1713 (K. Kölbl, München, 1964) (Foto: Departement Chemie und Angewandte Biowissenschaften der ETH Zürich).

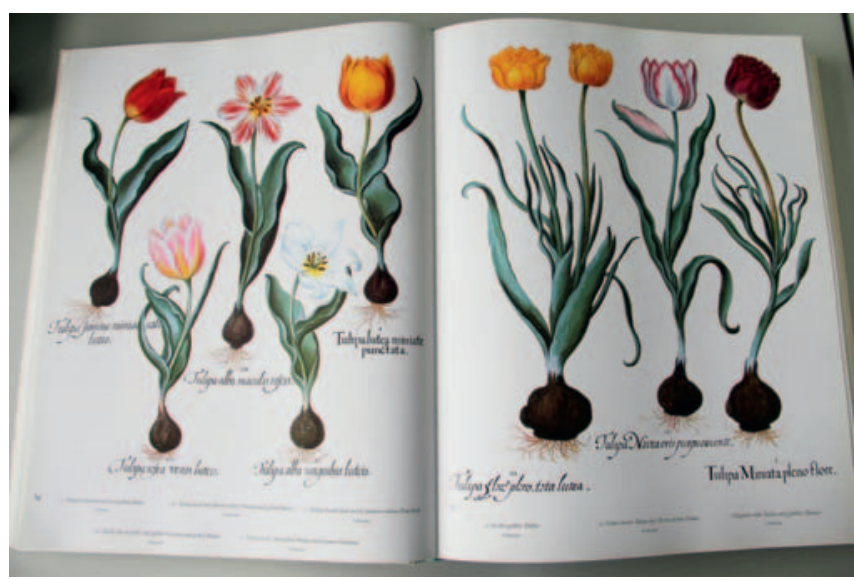

Abb. 5. «Der Garten von Eichstätt/Hortus Eystettensis - Das grosse Herbarium des Basilius Besler von 1613». Faksimile-Ausgabe, München, Schirmer/ Mosel Verlag, 1988/1997 (Foto: Departement Chemie und Angewandte Biowissenschaften der ETH Zürich).
Wochen lang blieb und Tulpenzwiebeln en masse erwarb. Der teuerste Preis für eine Tulpe betrug etwa 40 Gulden, wofür eine Waschfrau 2 Jahre in markgräflichen Diensten hätte arbeiten müssen.

In seinem Karlsruher Schlossgarten wurden 1733 fast 5000 Tulpenarten registriert, bei vier Arten zwischen 10000 und 84000 Stück. Auch im Garten seines Herrenhauses in Basel, dem Markgräflerhof, blühten Tausende von Tulpen.

Weil ihn die Schönheit der insbesondere «elegantissimae species» der Tulpe so faszinierte, liess der Markgraf wie andere Fürsten auch die Besonderheit jeder einzelnen Art und Sorte im Bild festhalten. Es entstanden 20 Folianten (von diesen sind heute noch zwei vorhanden) mit etwa 6000 Pflanzenaquarellen, auf denen rund 5300 Tulpen dargestellt waren [12, 13].

In den Jahren 1727 und 1728 war im Karlsruher Garten übrigens ein junger Mann beschäftigt, der mit seinen Tulpen- und Hyazinthenbildern grossen Eindruck auf den Markgrafen machte. Es handelte sich um den später berühmten Blumenmaler Georg Dionys Ehret ${ }^{*} 1708$ in Heidelberg), der in der übernächsten Ausgabe der SCHWEIZERISCHEN ZeITSCHRIFT FÜr GANZHEITSMEDIZIN nochmals erwähnt wird. Denn ausser von Clusius und Besler besass auch der Pharmazeut Prof. Carl Hartwich (1851-1917) (siehe auch Beitrag in Heft 2/14) von diesem einige Druckwerke in seiner Büchersammlung, die Anlass dieser Artikelserie ist.

\section{Literatur}

1 www.augsburgwiki.de/index.php/Augsburg Wiki/AugsburgerTulpen.

2 http://de.wikipedia.org/wiki/Ogier_Ghislain_ de_Busbecq.

3 Speta F: Auf den Spuren von Scilla Amoena. Naturkundliches Jahrbuch der Stadt Linz. 1976;22:73-102. www.landesmuseum.at/pdf frei_remote/NKJB_22_0073-0102.pdf.

4 http://de.wikipedia.org/wiki/Tulpenmanie.

5 von Petersdorff W: Eine Blumenzwiebel für 87.000 Euro. Frankfurter Allgemeine Zeitung, 18.03.2008, Nr. 66, p. 21.www.faz.net/ aktuell/finanzen/fonds-mehr/historischefinanzkrisen-niederlande-1637-eine-blumenzwiebel-fuer-87-000-euro-1283731-p2. html?printPagedArticle=true\#pageIndex_2.

6 http://de.wikipedia.org/wiki/Johann_Konrad_ von Gemmingen.

7 http://de.wikipedia.org/wiki/Basilius_Besler. 8 http://de.wikipedia.org/wiki/Hortus_ Eystettensis.

9 www.deutsches-museum.de/bibliothek/ unsere-schaetze/biologie/besler/der-hortuseystettensis.
10 Hofmann M, Zöhl C: Hortus Eystettensis. Studien zur Entstehung des Kupferstichwerks und zum Exemplar des Andrea Vendramin. http://archiv.ub.uni-heidelberg.de/ artdok/volltexte/2010/963.

11 http://de.wikipedia.org/wiki/Karl_III. Wilhelm_(Baden-Durlach).

12 www.blb-karlsruhe.de/virt_bib/tulpen.

13 Digitale Sammlung der Badischen Landesbibliothek Karlsruhe: Karlsruher Tulpenbuch - Karlsruhe 3302. Karlsruhe, 1730. 